\title{
Material tailoring of the femoral component in a total knee replacement to reduce the problem of aseptic loosening
}

\begin{abstract}
Aseptic loosening of femoral components is a significant problem affecting the life of current total knee replacements. To help reduce the problem of aseptic loosening, a new metalceramic poros functionally graded biomaterial (FGBM) has been designed to replace the existing metal alloy material normally used. In order to investigate the effect of using a FGBM on distal femur stresses compared to using standard material in a femoral component, a three-dimensional finite element model of the knee prosthesis has been developed. The results of the modeling and subsequent analysis indicate that by using the new FGBM compared to the existing material in a femoral component, higher levels of stress can be realized in the adjacent bone area of the femur and as a consequence reduce harmful atrophy effects. Also, by a judicious choice of material combinations and variation of porosity in the FGBM, the surface properties can be tailored to improve wear resistance at the articular interface and bone anchorage at the femoral end, as well as varying the stiffness in the core of the femoral component. Therefore, the use of the new FGBM improves the performance of knee prostheses by addressing concurrently the three current leading causes of failure; stressshielding of the bone by the implant, wear of the articular surfaces, and the development of soft tissue at the bone/prosthesis interface as a result of relative implant motion.
\end{abstract}

Keyword: Materials selection; Functionally graded materials; Knee implant design; Finite element analysis 